\title{
Microsurgical Techniques for the Treatment of Breast Cancer-related Lymphedema: a Systematic Review
}

\author{
Tiara R. Lopez Penha, MD ${ }^{1,2}$ Charlotte IJsbrandy, MD ${ }^{2}$ Nicole A. M. Hendrix, MD² \\ Esther M. Heuts, MD, PhD ${ }^{1}$ Adri C. Voogd, $\mathrm{PhD}^{3}$ Maarten F. von Meyenfeldt, MD, $\mathrm{PhD}^{1}$
}

Rene R. W. J. van der Hulst, MD, PhD²

${ }^{1}$ Department of General Surgery, Maastricht University Medical Centre, Maastricht, The Netherlands

2 Department of Plastic, Reconstructive, and Hand Surgery, Maastricht University Medical Centre, Maastricht, The Netherlands

${ }^{3}$ Department of Epidemiology, Maastricht University, Maastricht, The Netherlands

J Reconstr Microsurg 2013;29:99-106.
Address for correspondence and reprint requests Tiara R. Lopez Penha, MD, Department of Plastic, Reconstructive, and Hand Surgery, Maastricht University Medical Centre, PO Box 5800, 6202 AZ

Maastricht, The Netherlands (e-mail: t.lopez@maastrichtuniversity.nl).

\begin{abstract}
Keywords

- lymphedema

- breast cancer

- lymphatic microsurgery

Background Upper limb lymphedema is one of the most underestimated and debilitating complications of breast cancer treatment. The aim of this review is to summarize the recent literature for evidence of the effectiveness of lymphatic microsurgery for the treatment of breast cancer-related lymphedema (BCRL).

Methods A search was conducted for articles published from January 2000 until January 2012. Only studies on secondary lymphedema after breast cancer treatment and those examining the effectiveness of microsurgery were included.

Results No randomized clinical trials or comparative studies were available. Ten caseseries met inclusion criteria: (composite) tissue transfer $(n=4)$, lymphatic vessel transfer $(n=2)$, and derivative microlymphatic surgery $(n=4)$. Limb volume/circumference reduction varied from 2 to $50 \%$ over a follow-up time ranging from 1 to 132 months. Postoperative discontinuation rates of conservative therapy were only reported after composite tissue transfer, ranging from 33 to $100 \%$ after 3 to 24 months. Clear selection criteria for lymphatic surgery and lymphatic flow assessment were absent in most studies.

Conclusion We identified important methodological shortcomings of the available literature. Evidence acquired through comparative studies with uniform patient selection is lacking. Consistent positive findings with regards to limb volume reduction and limited complications are reasons to further explore these techniques in methodologically superior studies.
\end{abstract}

Upper limb lymphedema is one of the most underestimated and debilitating complications of breast cancer treatment. Hitherto, lymphedema treatment options have mainly been based on the lifelong application of a combination of conservative techniques, such as compression garments/bandages and manual lymph drainage. ${ }^{1-3}$ Conservative therapy is initially aimed at alleviating symptoms without curative intent; with such treatment, some patients may achieve sufficient limb volume reduction and symptom relief. In cases of inadequate disease management, lymphedema may received

May 29, 2012

accepted

August 24, 2012

published online

December 19, 2012
Copyright ( $\odot 2013$ by Thieme Medical Publishers, Inc., 333 Seventh Avenue, New York, NY 10001, USA. Tel: +1(212) 584-4662.
DOI http://dx.doi.org/ 10.1055/s-0032-1329919. ISSN 0743-684X. 
progress: arm-swelling transforming from a predominantly fluid encompassing entity to fibrosis and fat. ${ }^{4,5}$ Lymphedema in its late chronic phase is irreversible and accompanied by more symptoms and physical impairments, consequently compromising the quality of survivorship. ${ }^{6-10}$ An alternative treatment option could be beneficial for patients that are not responsive to standard conservative therapy. Lymphatic microsurgery could be such a treatment option, if treatment would be applied before lymphedema reaches its chronic, irreversible phase.

\section{Derivative (Super) Microlymphatic Surgery}

Microlymphatic repair for lymphedematous limbs emerged with the development of reconstructive microsurgery in the late 1960s. The very first experimental studies reported were in obstructive canine models. In these studies, communications were created between the lymphatic and venous systems to divert static lymph fluid away from the obstruction site in a technique called lymphatic venous anastomoses. ${ }^{11}$ The canine studies reported low and inconsistent patency rates as the result of this intervention, triggering the further development of the derivative microlymphatic technique. Refinements were made to reduce the chances of venous backflow and, consequently, bypass stenosis. However, limitations in the available microsurgical technology hampered the long-term establishment of viable anastomoses, as the pressure gradient between the lymphatic and venous systems were still too high. Until the beginning of this millennium there was little progression in lymphatic surgical techniques. In the contemporary clinical field of microvascular surgery, anastomoses with vessels as small as $0.3 \mathrm{~mm}$ in diameter are possible; this is also known as "super microsurgery." Because of these technical refinements, it is now possible to create multiple bypasses between lymphatic (ranging from 0.3 to 0.5 $\mathrm{mm})$ and venular vessels $(0.5 \mathrm{~mm})$ found in the subdermal plane of a lymphedematous limb. Anastomosing smaller vessels of the two vascular systems is hypothesized to ensure better bypass patency due to the minor intravascular pressure differences. ${ }^{12}$ Over the years evidence has been reported of lymphatic repair being effective in patients with secondary lymphedema of both the upper and lower extremities using lymphatic venular anastomoses. ${ }^{13-15}$

\section{Tissue Transfer}

Another technique to improve lymphatic drainage is the vascularized lymph node transfer with or without simultaneous free flap reconstruction. ${ }^{16}$ Experimental studies on lymph node transplantation in small animal studies have reported promising results of lymphatic function restoration, and with that, the facilitation of lymphedema resolution. ${ }^{17,18}$ Moreover, an alternative approach is achieved through the interposition of autologous tissue (i.e., lymph vessel), in which an attempt is made to restore flow within a damaged lymphatic system by bypassing the site of blockage. The transplanted lymph vessel(s) can function as a bridge connecting the afferent and efferent lymphatic vessels from the obstruction site. ${ }^{19}$
The recent movement toward the incorporation of evidence-based medicine into plastic surgery ${ }^{20}$ has stressed the importance of good quality research as the core of the clinical decision-making process. The primary aim of this systematic review is to summarize the recent literature for evidence of the effectiveness of lymphatic microsurgery for the treatment of breast cancer-related lymphedema (BCRL). Specifically, the effect of surgery on upper-limb volume/circumference reduction, symptom relief, lymph flow improvement, and the discontinuation of compression therapy postoperatively will be evaluated.

\section{Methods}

\section{Literature Search}

An electronic search was conducted in MEDLINE and PubMed for literature published from January 2000 until January 2012. Three independent investigators (TLP, NH, CI) performed the search. The following search terms were used: lymphoedema, lymphedema, microsurgery, surgical treatment, breast cancer, lymph node transfer, lymphovenous anastomoses, lymphaticovenular anastomoses, and lymph vessel transplantation. The search was limited to English, Dutch, German, and French literature, and we also hand-searched reference lists of the relevant articles found. Abstracts were scanned for relevance. Only the studies providing data on patients with secondary lymphedema after breast cancer treatment and those examining the effectiveness of microsurgical intervention were eligible for inclusion. Data from studies on primary lymphedema, lower extremity lymphedema, and mixed groups of lower and upper extremity lymphedema were excluded. All articles were evaluated for duplicate reporting on the same patient population and if so removed.

\section{Data Extraction}

The following data were extracted if available: surgical intervention type (lymph node transplantation, lymph vessel transplantation, or derivative lymphatic surgery), size of the patient population, mean follow-up time, BCRL classification or stage, mean duration of BCRL before surgery, arm volume or circumference reduction, lymph flow improvement as quantified by lymphoscintigraphy, symptom relief (self-perceived pain, heaviness, and erysipelas), discontinuation of postoperative conservative therapy (compression garments/bandages or manual lymphatic drainage) and complications (donor or recipient site morbidity).

\section{Quality Assessment}

A detailed methodological quality assessment was conducted independently by three investigators (TLP, CI, NH). The studies were assessed using an 8-question checklist from The American Society of Plastic Surgeons (ASPS) for therapeutic studies. ${ }^{21}$ Each affirmative answer was a point. Thus, a total score of 8 was the maximum to be assigned per study. The quality scores were compared and disagreements resolved by consensus. 


\section{Data Analysis}

The median and range of the methodological quality rating of all the studies combined, as well as the studies per surgical technique, are provided. We compared the median quality ratings using the Mann-Whitney U test. A $p$ value $<0.05$ was considered significant. Data analysis was conducted using SPSS for Mac 17.0 (SPSS Inc. Chicago, IL, USA). As for pooling study results, this was not possible due to substantial clinical heterogeneity between the studies with respect to the outcomes of interest and the characteristics of the patient populations. As an alternative, we have therefore chosen to present the characteristics and primary outcomes of each study separately in a schematic overview, according to the microsurgical technique that was used.

\section{Results}

There were no randomized clinical trials or comparative studies available, thus we were limited to the inclusion of case series. A total of 19 case series were identified. Nine ${ }^{22-30}$ were excluded, as they described either lymphedema of only the lower extremity, ${ }^{22-25}$ a mix of lower and upper extremity lymphedema, ${ }^{26-29}$ or a mix of primary and secondary upper extremity lymphedema. ${ }^{30}$ The remaining 10 studies were included for assessment (-Table $\mathbf{1}$ ). In these studies, three types of microlymphatic surgery were described: composite tissue transfer, lymphatic vessel transfer, and derivative surgery. The number of patients per case series was small, ranging from 6 to 24 women. However, one study reported evidence on 127 women. ${ }^{35}$ Follow-up time varied amongst the studies, ranging from 5 to 72 months in articles evaluating derivative lymphatic surgery and 6 to 132 months in those evaluating (composite) tissue transfer. Different measures were used to assess treatment outcome. These included reduction of limb volume, ${ }^{35,36,38,39}$ circumference, ${ }^{31,33,34,37,40}$ or symptomatology $31,32,38-40$ and improvement of lymphatic function quantified by lympho- scintigraphy. $31,33,34,36,38-40$

\section{Quality Assessment}

The median methodological quality rating of the 10 studies was 4.5 (range 2 to 5 ) (-Table $\mathbf{1}$ ). The studies on (composite) tissue transfer had a higher median quality score compared with that of derivative techniques, respectively 5 (range 2 to 5 ) versus 4 (range 3 to 5 ). However, this difference was not statistically significant $(p=0.567)$.

\section{(Composite) Tissue Transfer}

A total of four retrospective case series $(n=52)$ on composite tissue transfer for the treatment of BCRL were identified (-Table 2). Three $(n=39)$ described a similar operative technique: lymph nodes harvested at the inguinal site with the vascular structures and fat surrounding the superficial circumflex iliac vessels set in the axilla of the affected limb using the thoracodorsal vessels as recipient vessels. ${ }^{32-34}$ of these three, Saaristo et al modified the technique by combining it with a deep inferior epigastric perforator/musclesparing transverse rectus abdominis muscle (DIEP/msTRAM)

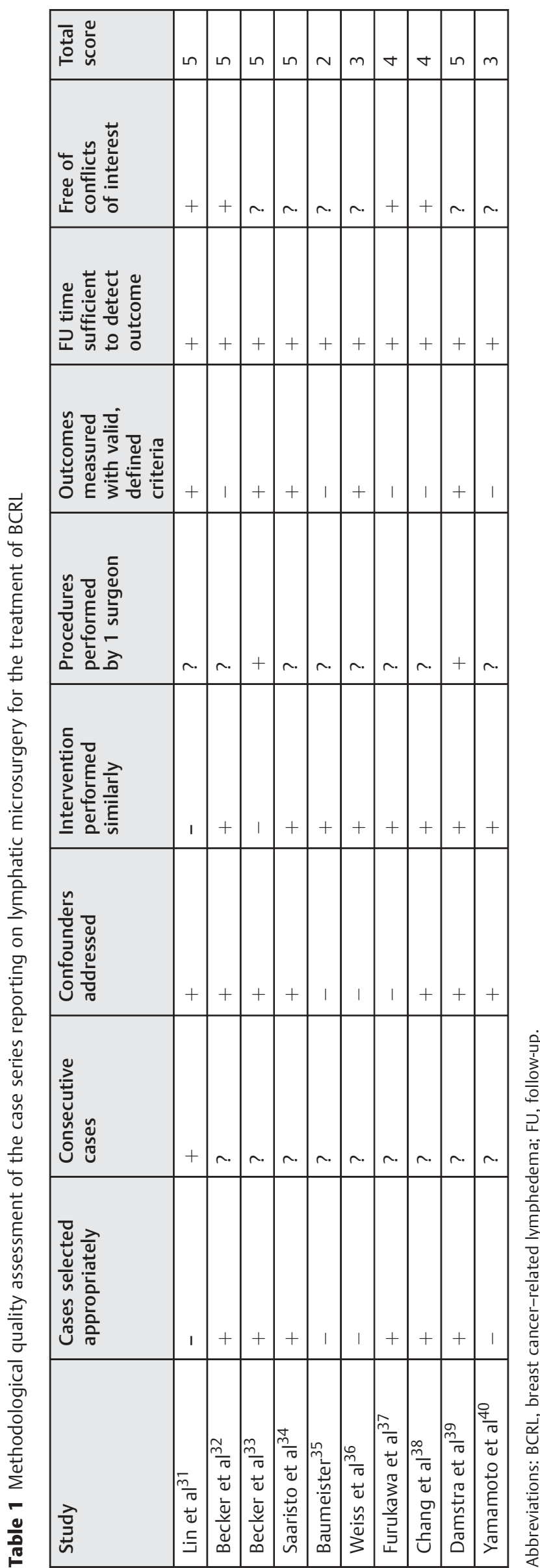




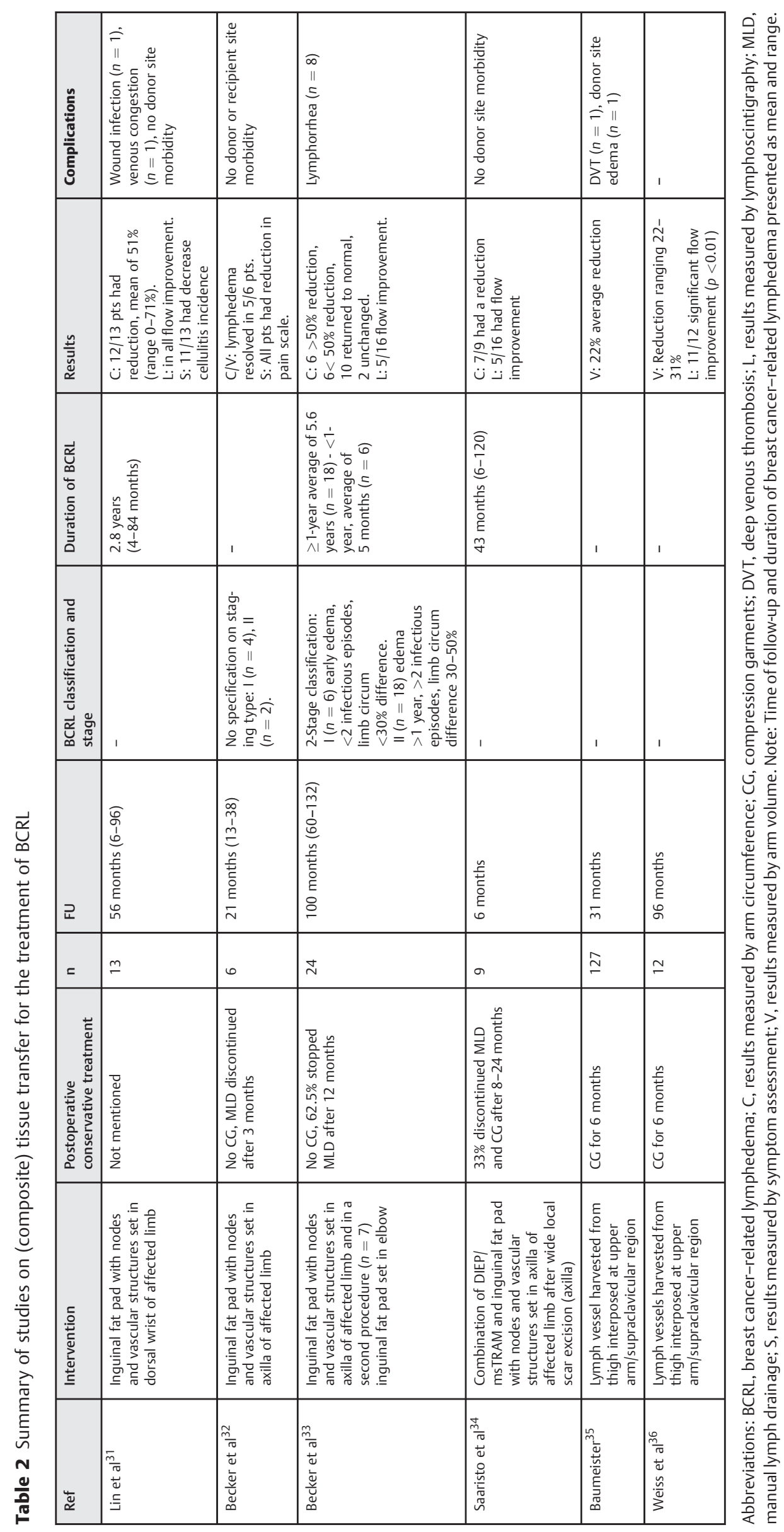


free flap for simultaneous breast reconstruction. The technique described by Lin et al differed from the other three in that the recipient site was the dorsal wrist of the affected arm instead of the axilla. Circumference reduction rate was provided by one study, ${ }^{31}$ stating an average reduction of $51 \%$ at 4.7 years follow-up. As for symptom improvement, Becker et al reported a reduction of pain in all 6 patients directly postoperatively, and Lin et al reported a reduction of the incidence of cellulitis in 11 of the 13 patients. Of the studies providing quantitative lymph flow assessment, improvement was reported in all three. ${ }^{31,33,34}$ Three studies reported the discontinuation rate of postoperative compression therapy, ranging from 33 to $100 \% 3$ months to 2 years after surgery. ${ }^{32-34}$ No donor site morbidity was reported.

Two studies $(n=139)$ were identified reporting on lymph vessel transplantation for the treatment of BCRL. The largest series $(n=127)$, a retrospective assessment by Baumeister et $\mathrm{al}$, reported an average volume reduction of $22 \%$ at a followup of 31 months. ${ }^{35}$ The rate of postoperative discontinuation of compression therapy was not addressed. As for postoperative complications, one case of donor site edema was reported.

\section{Derivative (Super) Microsurgical Techniques}

A total of four prospective case series $(n=47)$, evaluating derivative (super) microsurgery for the treatment of BCRL, met the inclusion criteria ( - Table 3 ). Three of the four studies reported a volume or circumference decrease greater than $30 \%$ at a follow-up time ranging from 5 to 72 months. ${ }^{37,38,40}$ Results were less favorable in a study by Damstra et al, reporting a limb volume reduction of $2 \%$ with accompanying unchanged lymphatic flow 12 months postoperatively. ${ }^{39}$ Three of the four studies showed improvement of symptomatic complaints postoperatively. ${ }^{38-40}$ Although only addressed by two studies, no postoperative complications were reported. ${ }^{38,40}$ Postoperative conservative therapy in the form of compression therapy and/or bandaging was continued indefinitely in all case series.

\section{Discussion}

In this review we summarized the available literature on lymphatic microsurgery for the treatment of BCRL. We were primarily interested in the efficacy of derivative lymphatic surgery and (composite) tissue transfer on upper-limb volume or circumference reduction, relief of symptoms related to BCRL, lymphatic function, and the discontinuation of conservative therapy postoperatively. Data could only be acquired through a mix of pro- and retrospective case series, as randomized or comparative studies were not available. This resulted in level IV evidence. With respect to the methodological quality, the studies on composite tissue transfer rated best when compared with that of lymph vessel transfer and derivative surgery (-Table $\mathbf{1}$ ). Two criteria were most often associated with a low methodological rating. The first is incomplete information on the patient selection procedure for surgery. As differentiating between early nonfibrotic lymphedema and chronic lymphedema is an important

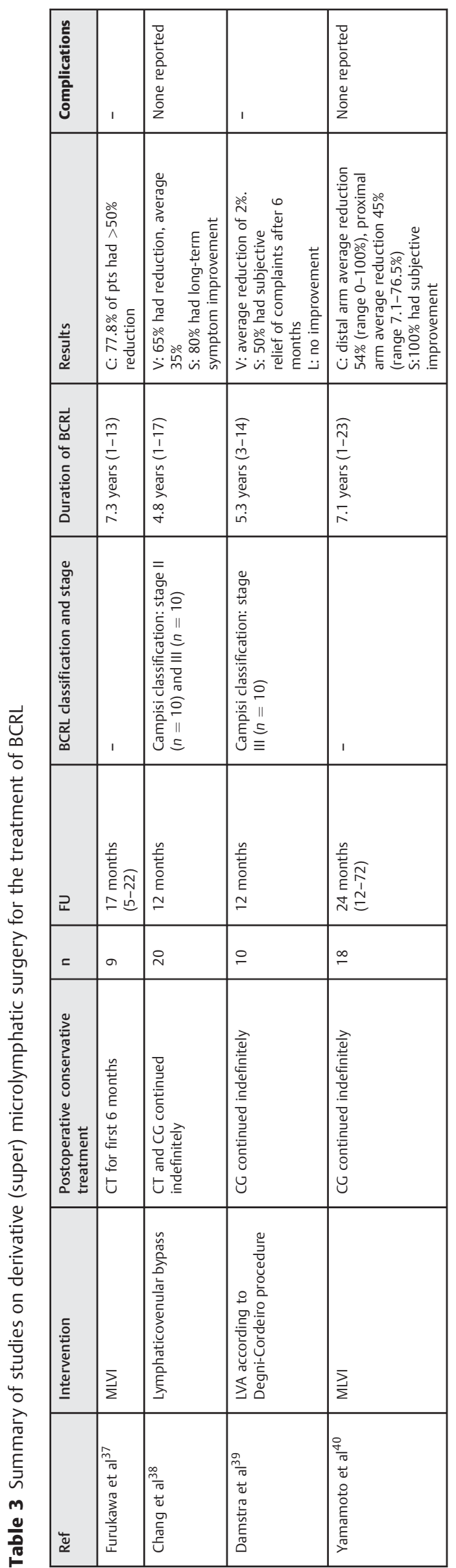


outcome-determining factor, the recording of these lymphedema stages when selecting study patients is essential. Second, the postsurgical outcome assessment should not only include limb volume or circumference measurements but should also be complemented with monitoring of lymphatic flow.

Unfortunately, it was not possible to pool the results of the different studies included in this review due to incomplete data documentation, but most importantly because of the large difference between the studies with respect to the criteria used for selecting patients for surgery. For example, only four studies stratified patients into lymphedema clinical stages before ensuing surgery, whereas the others did not take the pathophysiological nature of lymphedema into account, resulting in a heterogeneous patient population.

\section{Primary Outcomes}

All but one study ${ }^{39}$ noted consistent reduction of upper-limb volume or circumference reduction from surgical intervention through derivative lymphatic surgery, ${ }^{37,38,40}$ lymph node transplantation $^{31-34}$ or lymphatic vessel transfer. ${ }^{35,36}$ Damstra et al, reporting on results after lymphatic venous anastomosis, measured a disappointing mean volume difference of $2 \%$ after 1 year. There are, however, two aspects in the methodology of this study that might have affected the outcome. First, a surgical procedure according to Degni-Cordeiro was applied. This is a rather outdated technique originating from the early $1980 \mathrm{~s},{ }^{41} \mathrm{a}$ period in which derivative lymphatic surgery had proven low bypass patency due to the intravascular pressure gradient. Second, the selection criteria for the group of patients that would undergo derivative lymphatic surgery have affected the outcome of this study. It is stated by the authors that complete limb volume reduction was achieved in nearly half of the patient population when circumferential suction-assisted lipectomy was performed after the initial lymphatico-venous anastomosis (LVA) operation. This suggests a chronic lymphedema stage. The natural progression of lymphedema sees to the change of limb swelling composition, from an at first predominantly fluidcontaining entity to the addition of fibrosis, fat, and protein. So, even if the static lymph fluid is relieved, swelling still remains in the form of excess fat and fibrosis. ${ }^{42,43}$ Furthermore, chronic lymphedema also suggests a prolonged hypertensive state within the lymphatic system. This phenomenon causes irreversible damage to the lymph vessels (i.e., degeneration of smooth muscle cells), ${ }^{44,45}$ rendering the lymph vessels incapable of lymph fluid propulsion. Therefore, failure is inevitable in the attempts of producing patent lymphatic venous bypasses.

Aside from limb volume or circumference reduction, surgery effectiveness can be determined by lymphatic function. Lymphoscintigraphy can visualize lymphatic flow, providing qualitative information on lymph transport in the affected limb. Only half the studies $31,33,34,36,39$ assessed in this review evaluated the effect of lymphatic microsurgery on the lymphatic function. This is a major methodological flaw, as the ultimate aim of every lymphatic surgery should be to repair the function of the damaged lymphatic system. Moreover, in derivative surgery it is important to monitor lymphaticvenular bypass patency. Until recently there were no means of doing so. The use of indocyanine green fluorescence lymphangiography appears promising in this respect. ${ }^{46,47}$

The effect of surgery on symptom relief was not a main focus for many of the studies in this review. A specific decrease in neuropathic pain and in cellulitis rates was reported in two small populations after lymph node transplantation. ${ }^{31,32}$ As for the effect of derivative lymphatic surgery, a subjective relief of symptoms was noted in 50 to $100 \%$ of the patients in three studies. ${ }^{38-40}$

The discontinuation of postoperative conservative therapy was realized, although in variable rates, 3 to 24 months after surgery in the three studies evaluating inguinal lymph node transfer to the axilla of the lymph edematous arm. ${ }^{32-34}$ As reported, ${ }^{31,33}$ the results were better when the duration of lymphedema was the shortest before lymph node transfer, suggesting that this surgery cannot only be used as an alternative treatment for conservative therapy-resistant BCRL, but also as a curative up-front approach for lymphedema.

Even though we were limited to level IV evidence, a preference can be given to the inguinal lymph node transfer based on the reviewed literature. Not only was this the only technique that made the discontinuation of postoperative conservative therapy possible, it was also the technique described in better methodological quality studies compared with that of the other techniques. Furthermore, because this surgical procedure can be combined with autologous breast reconstruction, it might be easier to incorporate it into current breast cancer management programs.

\section{Conclusion}

An overview is presented of the current evidence base for the effectiveness of lymphatic microsurgery for the treatment of BCRL. We have identified important methodological shortcomings of the available literature. Evidence acquired through comparative prospective studies with uniform patient selection is lacking. The consistent positive findings with regard to limb volume/circumference reduction, and limited to no complications reported after microlymphatic surgery are, however, reasons to further explore these techniques in methodologically superior studies, perhaps answering the question as to when is the most appropriate time in the disease process for lymphatic surgery. We believe that the true contribution of microlymphatic surgery for the treatment of BCRL will only be elucidated through protocolized treatment initiation by experienced microsurgeons. In our institution we are currently in the process of setting up a prospective study: an integrated care program in which breast cancer patients are prospectively screened for lymphedema and are structurally treated with conservative therapy followed by lymph node transplantation or derivative surgery in conservative treatment-resistant cases.

\section{References}

1 Kligman L, Wong RK, Johnston M, Laetsch NS. The treatment of lymphedema related to breast cancer: a systematic review and evidence summary. Support Care Cancer 2004;12:421-431 
2 Moseley AL, Carati CJ, Piller NB. A systematic review of common conservative therapies for arm lymphoedema secondary to breast cancer treatment. Ann Oncol 2007;18:639-646

3 McNeely ML, Peddle CJ, Yurick JL, Dayes IS, Mackey JR. Conservative and dietary interventions for cancer-related lymphedema: a systematic review and meta-analysis. Cancer 2011;117: 1136-1148

4 International Society of Lymphology. The diagnosis and treatment of peripheral lymphedema. 2009 Concensus Document of the International Society of Lymphology. Lymphology 2009;42:51-60

5 Ji RC. Lymphatic endothelial cells, lymphedematous lymphangiogenesis, and molecular control of edema formation. Lymphat Res Biol 2008;6:123-137

6 Rietman JS, Dijkstra PU, Debreczeni R, Geertzen JH, Robinson DP, De Vries J. Impairments, disabilities and health related quality of life after treatment for breast cancer: a follow-up study 2.7 years after surgery. Disabil Rehabil 2004;26:78-84

7 Morgan PA, Franks PJ, Moffatt CJ. Health-related quality of life with lymphoedema: a review of the literature. Int Wound J 2005;2: 47-62

8 Ahmed RL, Prizment A, Lazovich D, Schmitz KH, Folsom AR. Lymphedema and quality of life in breast cancer survivors: the Iowa Women's Health Study. J Clin Oncol 2008;26:5689-5696

9 Oliveri JM, Day JM, Alfano CM, et al. Arm/hand swelling and perceived functioning among breast cancer survivors 12 years post-diagnosis: CALGB 79804. J Cancer Surviv 2008;2:233-242

10 Nesvold IL, Reinertsen KV, Fosså SD, Dahl AA. The relation between arm/shoulder problems and quality of life in breast cancer survivors: a cross-sectional and longitudinal study. J Cancer Surviv 2011;5:62-72

11 O'Brien BM. Replantation and reconstructive microvascular surgery. Part II. Ann R Coll Surg Engl 1976;58:171-182

12 Nagase T, Gonda K, Inoue K, et al. Treatment of lymphedema with lymphaticovenular anastomoses. Int J Clin Oncol 2005;10: 304-310

13 Koshima I, Kawada S, Moriguchi T, Kajiwara Y. Ultrastructural observations of lymphatic vessels in lymphedema in human extremities. Plast Reconstr Surg 1996;97:397-405, discussion 406-407

14 O'Brien BM, Mellow CG, Khazanchi RK, Dvir E, Kumar V, Pederson WC. Long-term results after microlymphaticovenous anastomoses for the treatment of obstructive lymphedema. Plast Reconstr Surg 1990;85:562-572

15 Campisi C, Boccardo F. Role of microsurgery in the management of lymphoedema. Int Angiol 1999;18:47-51

16 Massey MF, Spiegel AJ, Levine JL, et al; Group for the Advancement of Breast Reconstruction. Perforator flaps: recent experience, current trends, and future directions based on 3974 microsurgical breast reconstructions. Plast Reconstr Surg 2009;124:737-751

17 Blum KS, Hadamitzky C, Gratz KF, Pabst R. Effects of autotransplanted lymph node fragments on the lymphatic system in the pig model. Breast Cancer Res Treat 2010;120:59-66

18 Tobbia D, Semple J, Baker A, Dumont D, Johnston M. Experimental assessment of autologous lymph node transplantation as treatment of postsurgical lymphedema. Plast Reconstr Surg 2009;124: 777-786

19 Baumeister RG, Siuda S. Treatment of lymphedemas by microsurgical lymphatic grafting: what is proved? Plast Reconstr Surg 1990;85:64-74, discussion 75-76

20 Rohrich RJ, Eaves FF III. So you want to be an evidence-based plastic surgeon? A lifelong journey. Plast Reconstr Surg 2011;127: 467-472

21 Sullivan D, Chung KC, Eaves FF III, Rohrich RJ. The level of evidence pyramid: indicating levels of evidence in Plastic and Reconstructive Surgery articles. Plast Reconstr Surg 2011;128:311-314

22 Maegawa J, Mikami T, Yamamoto Y, Satake T, Kobayashi S. Types of lymphoscintigraphy and indications for lymphaticovenous anastomosis. Microsurgery 2010;30:437-442
23 Demirtas Y, Ozturk N, Yapici O, Topalan M. Supermicrosurgical lymphaticovenular anastomosis and lymphaticovenous implantation for treatment of unilateral lower extremity lymphedema. Microsurgery 2009;29:609-618

24 Matsubara S, Sakuda H, Nakaema M, Kuniyoshi Y. Long-term results of microscopic lymphatic vessel-isolated vein anastomosis for secondary lymphedema of the lower extremities. Surg Today 2006;36:859-864

25 Koshima I, Nanba Y, Tsutsui T, Takahashi Y, Itoh S. Long-term follow-up after lymphaticovenular anastomosis for lymphedema in the leg. J Reconstr Microsurg 2003;19:209-215

26 Narushima M, Mihara M, Yamamoto Y, Iida T, Koshima I, Mundinger GS. The intravascular stenting method for treatment of extremity lymphedema with multiconfiguration lymphaticovenous anastomoses. Plast Reconstr Surg 2010;125:935-943

27 Lee BB, Laredo J, Neville R. Reconstructive surgery for chronic lymphedema: a viable option, but. Vascular 2011;19:195-205

28 Campisi C, Bellini C, Campisi C, Accogli S, Bonioli E, Boccardo F. Microsurgery for lymphedema: clinical research and long-term results. Microsurgery 2010;30:256-260

29 Auba C, Marre D, Rodríguez-Losada G, Hontanilla B. Lymphaticovenular anastomoses for lymphedema treatment: 18 months postoperative outcomes. Microsurgery 2012;32:261-268

30 Koshima I, Inagawa K, Urushibara K, Moriguchi T. Supermicrosurgical lymphaticovenular anastomosis for the treatment of lymphedema in the upper extremities. J Reconstr Microsurg 2000;16: 437-442

31 Lin CH, Ali R, Chen SC, et al. Vascularized groin lymph node transfer using the wrist as a recipient site for management of postmastectomy upper extremity lymphedema. Plast Reconstr Surg 2009;123:1265-1275

32 Becker C, Pham DN, Assouad J, Badia A, Foucault C, Riquet M. Postmastectomy neuropathic pain: results of microsurgical lymph nodes transplantation. Breast 2008;17:472-476

33 Becker C, Assouad J, Riquet M, Hidden G. Postmastectomy lymphedema: long-term results following microsurgical lymph node transplantation. Ann Surg 2006;243:313-315

34 Saaristo AM, Niemi TS, Viitanen TP, Tervala TV, Hartiala P, Suominen EA. Microvascular breast reconstruction and lymph node transfer for postmastectomy lymphedema patients. Ann Surg 2012;255:468-473

35 Baumeister RG, Frick A. [The microsurgical lymph vessel transplantation]. Handchir Mikrochir Plast Chir 2003;35:202-209

36 Weiss M, Baumeister RG, Hahn K. Post-therapeutic lymphedema: scintigraphy before and after autologous lymph vessel transplantation: 8 years of long-term follow-up. Clin Nucl Med 2002;27: 788-792

37 Furukawa H, Osawa M, Saito A, et al. Microsurgical lymphaticovenous implantation targeting dermal lymphatic backflow using indocyanine green fluorescence lymphography in the treatment of postmastectomy lymphedema. Plast Reconstr Surg 2011;127: 1804-1811

38 Chang DW. Lymphaticovenular bypass for lymphedema management in breast cancer patients: a prospective study. Plast Reconstr Surg 2010;126:752-758

39 Damstra RJ, Voesten HG, van Schelven WD, van der Lei B. Lymphatic venous anastomosis (LVA) for treatment of secondary arm lymphedema. A prospective study of 11 LVA procedures in 10 patients with breast cancer related lymphedema and a critical review of the literature. Breast Cancer Res Treat 2009;113: 199-206

40 Yamamoto Y, Horiuchi K, Sasaki S, et al. Follow-up study of upper limb lymphedema patients treated by microsurgical lymphaticovenous implantation (MLVI) combined with compression therapy. Microsurgery 2003;23:21-26

41 Degni M. New microsurgical technique of lymphatico-venous anastomosis for the treatment of lymphedema. Lymphology 1981;14:61-63 
42 Brorson H. From lymph to fat: complete reduction of lymphoedema. Phlebology 2010;25(Suppl 1):52-63

43 Brorson H, Svensson H. Liposuction combined with controlled compression therapy reduces arm lymphedema more effectively than controlled compression therapy alone. Plast Reconstr Surg 1998;102:1058-1067, discussion 1068

44 Koshima I, Kawada S, Moriguchi T, Kajiwara Y. Ultrastructural observations of lymphatic vessels in lymphedema in human extremities. Plast Reconstr Surg 1996;97:397-405, discussion 406-407
45 Nagase T, Gonda K, Inoue K, et al. Treatment of lymphedema with lymphaticovenular anastomoses. Int J Clin Oncol 2005;10: 304-310

46 Maegawa J, Yabuki Y, Tomoeda H, Hosono M, Yasumura K. Outcomes of lymphaticovenous side-to-end anastomosis in peripheral lymphedema. J Vasc Surg 2012;55:753-760

47 Mukenge SM, Catena M, Negrini D, et al. Assessment and follow-up of patency after lymphovenous microsurgery for treatment of secondary lymphedema in external male genital organs. Eur Urol 2011;60:1114-1119 